\title{
Gas Source Localization Strategies for Teleoperated Mobile Robots. An Experimental Analysis
}

\author{
Andres Gongora, Javier Monroy, and Javier Gonzalez-Jimenez
}

\begin{abstract}
Gas source localization (GSL) is one of the most important and direct applications of a gas sensitive mobile robot, and consists in searching for one or multiple volatile emission sources with a mobile robot that has improved sensing capabilities (i.e. olfaction, wind flow, etc.). This work adresses GSL by employing a teleoperated mobile robot, and focuses on which search strategy is the most suitable for this teleoperated approach. Four different search strategies, namely chemotaxis, anemotaxis, gas-mapping, and visual-aided search, are analyzed and evaluated according to a set of proposed indicators (e.g. accuracy, efficiency, success rate, etc.) to determine the most suitable one for a human-teleoperated mobile robot. Experimental validation is carried out employing a large dataset composed of over 150 trials where volunteer operators had to locate a gas-leak in a virtual environment under various and realistic environmental conditions (i.e. different wind flow patterns and gas source locations). We report different findings, from which we highlight that, against intuition, visual-aided search is not always the best strategy, but depends on the environmental conditions and the operator's ability to understand how gas distributes.
\end{abstract}

\section{INTRODUCTION}

Robot teleoperation (also called telerobotics) is the remote operation of a robot to perceive and interact with the world at a distance [1]. A particular case is that of teleoperating a mobile robot, for instance, to work in hazardous environments (e.g. remote bomb disarming [2]), or to inspect difficult to reach sites (e.g collapsed buildings [3]) among others. In this context, another interesting application is the localization of volatile chemical sources, commonly addressed in literature as gas source localization (GSL). Specifically, the use of a teleoperated gas-sensitive mobile robot to remotely locate one or multiple gas emission sources.

Traditionally, GSL has been tackled with autonomous mobile robots in an attempt to automate the search process. Different approaches, ranging from bio-inspired techniques [4] to engineering solutions [5] have been proposed. Yet, due to the still limited capabilities of autonomous robots and the complex mechanism that rule gas dispersion [6], most works in this field have only been validated under laboratory conditions (i.e. unidirectional and laminar wind fields [7], absence of obstacles in the environment [8], etc.) far from the complexity of real-world settings. Hereof, a teleoperation

This work has been funded by the Governments of Spain and Andalusia, and the European Regional Development Fund under project TEP530.

Andres Gongora, Javier Monroy, and Javier Gonzalez-Jimenez are with with the Machine Perception and Intelligent Robotics (MAPIR) research group, University of Malaga, Spain.

andresgongora@uma.es

jgmonroy@uma.es

javiergonzalez@uma.es approach, which alleviates these drawbacks by introducing the human intuition and reasoning, seems a natural candidate for solution. An important issue to address in this case is the "definition" of the teleoperation interface. More concretely, what information needs to be provided to the operator for a successful and efficient resolution. Most certainly, it will involve gas identity and concentration measurements of the gas that is being tracked, which could be provided by carrying an electronic nose [9] (e-nose) on the robot. But the sense of smell alone might not always suffice, meaning that other sensory inputs, like the wind flow or visual clues, may also be needed to maximize the search performance. In this work we analyze the influence of these sensory inputs for olfactory telerobotics, specifically, those that match the following GSL approaches:

- Chemotaxis: it relies solely on chemical measurements from an e-nose to find the emission source, typically by travelling along the gradient of sensed gas towards where the concentration is highest [7], [10]. It has the advantage of being the simplest approach, but performs poorly for low concentration gas profiles or in turbulent environments [11].

- Anemotaxis: in addition to an e-nose, the robot is equipped with an anemometer to track the airborne gas plume [12]. Algorithms implementing this strategy commonly receive the name of "plume tracking methods", and involve following the sensed gas up-wind to its origin [8], [13], [14].

- Gas Mapping: it relies on spatio-temporal memory of the aforementioned variables to create a map of how the gases distribute in the environment [15], [16] from which to infer the location of the source [17]. This approach performs well in extremely unstructured and disordered airflow fields, where plumes of well-defined shapes are not likely to be formed. In such cases, mapping the gases of the entire inspection area might be the most reliable way to find the source, although certainly not the most efficient.

- Visual-aided search: this approach encompasses any GSL strategy that exploits prior knowledge about the appearance of the gas source to improve the search process [18]-[20]. This entails two important aspects, the proper recognition of objects in the scene, and the semantic inference to correlate the shape of an object with its smell and vice versa. Both of which should pose no challenge to a human operator. 


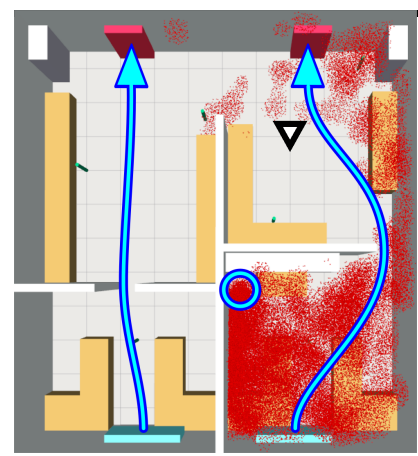

(a) Scenario 1

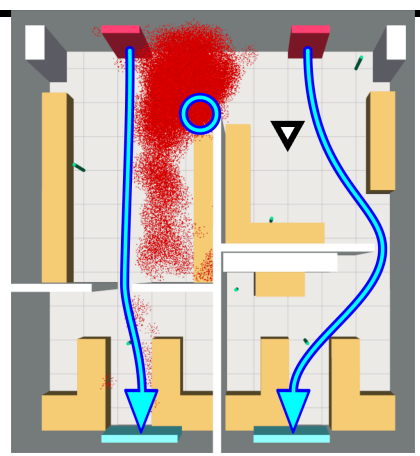

(b) Scenario 2

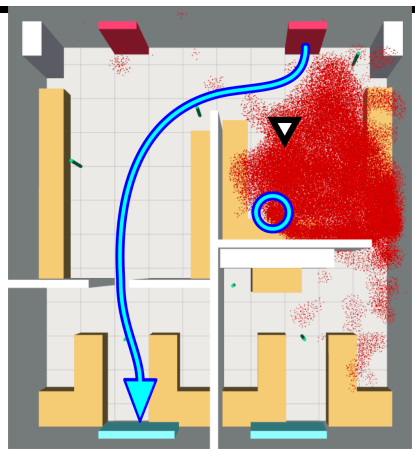

(c) Scenario 3

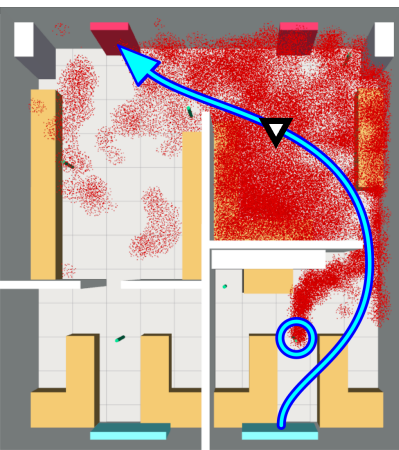

(d) Scenario 4

Fig. 1: The four scenarios of the simulated experiments showing the wind's main flow (arrows), the location of the active gas-leak (circles), other gas-leak candidates shown during visual-aided search (green cylinders), the distribution of the emitted gas after 60 seconds (red point-cloud), and the robot's starting position (black triangle).

The aim of this work is then to seek which of the above strategies is the most suitable for teleoperated GSL tasks. In pursuit of a fair comparison, we propose evaluating their average effectiveness and accuracy, how they affect the operator's search behaviour, and in terms of expended energy, their efficiency. The analysis is conducted on an extensive dataset composed over 150 experiments, gathered during a previous campaign [21], where users had to locate a gas source in a simulated environment with a teleoperated mobile robot. More specifically, and in accordance with the aforementioned GSL strategies, the experiments are classified in four different configurations: (i) pure chemotaxis, (ii) anemotaxis, (iii) gas mapping with chemical and wind flow data, and (iv) the latter plus visual clues (i.e. all sensors combined).

Next, Section II provides a description of the experimental setup and the collected dataset, and Section III proposes different magnitudes to compare the studied GSL strategies. Then, Section IV presents the results and discussion about our study, and finally, Section V offers the most relevant conclusions and suggests future research.

\section{ACQUisition OF THE EXPERIMENTAL DATA}

In this study we use a dataset for teleoperated GSL composed of more than 150 experiments presented in a previous work [21], which is briefly described next to make this paper self contained.

Experiments were performed in a simulated environment where volunteer participants had to locate as accurately as possible a gas source. The environment and the mobile platform were simulated within $\operatorname{ROS}^{1}$, and to obtain a realistic gas dispersion of the emitted volatiles, we employed a custom 3D CFD-based ${ }^{2}$ gas simulator. Four realistic and dynamic gas release setups were considered by varying the wind flow conditions and the source location (see Fig. 1). Users controlled the mobile platform via a web-based interface [22], [23], declaring the gas source by terminating the

\footnotetext{
${ }^{1}$ http://www.ros.org/

${ }^{2}$ http://www.openfoam.com/
}

experiment at the desired location.

Simulation trials were chosen instead of real-world experiments because it allowed us to repeat an experiment several times under identical test conditions (wind flow, gas release, source location, etc.), something hardly achievable in realworld scenarios [24]. Nevertheless, we must stress out that the data gathered during the experiments, and exploited along this study, was not that of the simulator, but those parameters and variables related to the user activity, including the search path, navigation commands and the execution time.

Finally, related to the different sensors and algorithms involved in the four GSL strategies to be analyzed, we simulated a photo ionization gas detector (PID), a 2D ultrasonic anemometer for wind flow sensing, implemented the GMRF gas distribution mapping algorithm [15], and visually displayed gas source candidates as green cylinders.

\section{iII. Evaluation Methodology}

Direct comparison between the different GSL strategies is not a trivial task because of the differences in the test scenarios and in the environmental conditions contained in the dataset. That is, one of the tested strategies could be advantageous when dealing with a certain type of gas distribution, yet fail for the others. Moreover, the evaluation criteria selected for such comparison has also a strong influence in the results. Notice that basic magnitudes like the robot's final distance to the source [25], or the time spent [12] might not be indicative of true performance differences when comparing GSL strategies under different environmental conditions.

In this work we analyze each combination of scenario and GSL strategy separately, and propose three indices to compare them:

\section{A. Success Ratio (S)}

We evaluate the success of a GSL trial by measuring the distance $(d)$ between the user estimated source location (the final position of the robot), and its actual location. A threshold distance $D_{t h}$ of $50 \mathrm{~cm}$ is set (also used by 
Authors' accepted manuscript: European Conference on Mobile Robotics (ECMR), 2017.

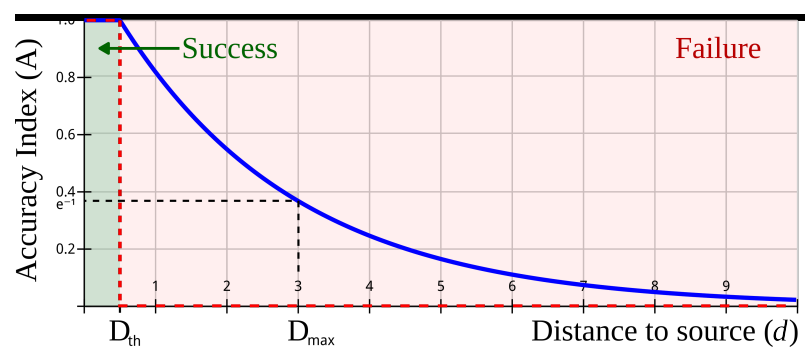

Fig. 2: Comparison between Accuracy Index (blue-solid line) and the success/failure binary label (red-dashed line) for increasing distances to the emission source $(d)$. For both indices $D t h=0.5 \mathrm{~m}$ and $D \max =3 \mathrm{~m}$.

other works [25], [26]) to label an experiment as successful $\left(d \leq D_{t h}\right)$ or not $\left(d>D_{t h}\right)$.

Based on this measure, we calculate the success ratio $(S)$ for each strategy as the ratio between successful attempts and the total number of trials [27].

\section{B. Accuracy Index $(A)$}

Although the success ratio gives us an insight into the average effectiveness of each strategy, it is too strict to assess its accuracy properly. For example, it does not distinguish between an experiment with a final error close to the specified $D_{t h}$, to another with a very large error (in any case both are marked as failures). For that reason we also compute a continuous accuracy index based on the final distance to the source as:

$$
A= \begin{cases}1 & \text { if } d \leq D_{t h} \\ e^{\frac{D_{t h}-d}{D_{\max }-D_{t h}}} & \text { otherwise }\end{cases}
$$

where $D_{\max }$ is a constant that controls the decrease rate by establishing a distance upon which the localization is considered to have failed. For our particular scenarios we chose $D_{\max }=3 \mathrm{~m}$, as greater distances mean that the user most probably declared the source location at the wrong room.

Note that $A$ is a better indicator of search accuracy than $d$ alone because it penalizes exponentially unsuccessful experiments instead of discarding them (see Fig. 2), and because can be adjusted to compensate for the environment dimensions.

\section{Efficiency Index $(\eta)$}

Besides accuracy, efficiency is the second most important aspect to consider when developing a GSL system for mobile robots. Because the energy a robot consumes depends on the duration of the experiment and the traveled distance, we must also evaluate how much the operators' path differ from optimal, understanding as optimal path the shortest possible one that goes from the starting position directly to the gasleak (accounting for obstacles), at the robot's maximum (safe) speed.

To measure efficiency, we use Eq. (2) as proposed by Hayes et al. [27]:

$$
\eta=\frac{E_{\text {min }}}{E}=\frac{\alpha T_{\min }+\beta P_{\text {min }}}{\alpha t+\beta p}
$$

which can be interpreted as the relation between the minimum required energy to locate the source (e.g. shortest possible path $P_{\min }$ and time $T_{\min }$ ) with the energy $E$ employed during the trial, where $\alpha$ and $\beta$ denote the energy cost per unit of employed time $(t)$ and travelled path length $(p)$, respectively. Hence, the efficiency index $\eta$ is a dimensionless quantity defined so that $\eta \in[0,1]$, with 1 being the optimal path solution.

Note that although $\alpha$ and $\beta$ can take any positive real value, we follow Hayes et al.'s suggestion and set them such that the minimum distance and time energy costs are equal, that is, $\alpha T_{\min }=\beta P_{\min }$. Also, because $T_{\min }$ is determined by the maximum safe operating speed $V_{\max }$ at which the robot can travel the path $P_{\min }$ in a specific environment, we know that $T_{\text {min }}=P_{\min } / V_{\max }$. Introducing both considerations into Eq. (2) we get:

$$
\eta=\frac{2 P_{\min }}{V_{\max } \cdot t+p}
$$

\section{Average Occupation Map}

Finally, and despite not being a quantifiable magnitude as such, we generate heat maps displaying the proportion of time the users spent, in average, at each location of the environment. These maps facilitate judging the influence of each strategy on the user's search behaviour to determine, for example, whether anemotaxis allowed to discard the exploration of rooms that exhausted clean air.

\section{ANALYSIS AND Discussion}

In this section we present the results of comparing each of the four GSL strategies according to the aforementioned indices.

\section{A. Search Success}

Table I summarizes the success ratios for all combinations of search strategy and scenario, as well as the total successes separately. Keep in mind that an experiment counts only as successful if the operator got within $50 \mathrm{~cm}$ of the emission source (refer to Section III-A).

\begin{tabular}{|c|c|c|c|c|c|}
\hline & chen & $\begin{array}{l}\text { xis } \\
\text { Aner }\end{array}$ & 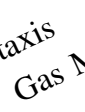 & $\begin{array}{l}\text { ping } \\
\text { visual }\end{array}$ & summinary \\
\hline Scen & 22.2 & 12.5 & 11.1 & 87.5 & 32.4 \\
\hline Scenario 2 & 22.2 & 37.5 & 22.2 & 90.9 & 45.9 \\
\hline Scenario 3 & 11.1 & 0.0 & 0.0 & 100.0 & 28.9 \\
\hline Scenario 4 & 27.3 & 60.0 & 38.5 & 50.0 & 42.9 \\
\hline All Scenarios & 21.1 & 28.6 & 19.5 & 83.8 & 37.8 \\
\hline
\end{tabular}

TABLE I: Percentual success ratios $(S)$ of the tested GSL strategies, individual and overal results for each scenario. 
Authors' accepted manuscript: European Conference on Mobile Robotics (ECMR), 2017.
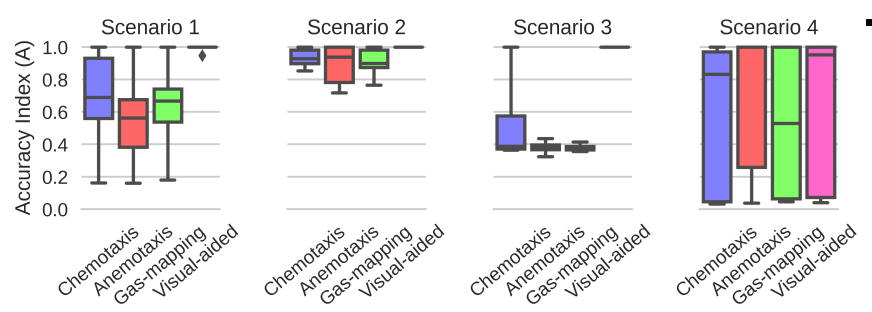

Fig. 3: Accuracy Index for each scenario and GSL strategy $($ Dth $=0.5 \mathrm{~m}$ and Dmax $=3 \mathrm{~m})$. This index represents how close the operator got to the emission source.

Visual-aided search has generally the best success ratio of them all. However, $S$ drops to $50 \%$ for Scenario 4, which, as we will later discuss in Section IV-D, might be because visual clues can be confusing if the environment contains very complex gas distributions. In this case, anemotaxis or gas-mapping appear more advantageous despite their usually low effectiveness.

As for chemotaxis, it does not seem to be specially successful for any of the considered scenarios. Still, it is a robust option with consistent results, even in Scenario 3 where anemotaxis and gas-mapping failed completely.

\section{B. Search Accuracy}

As mentioned in Section III-B, a more descriptive and fair comparison involves the use of the Accuracy Index (A). As can be observed in Fig. 3, accuracy retains some similarities with the success ratio (e.g. general effectiveness for each scenario), but now also reveals information about those experiments that failed locating the emission source.

Visual aided search remains in general the most accurate strategy, while anemotaxis and gas-mapping stay approximately on par to each other with similar medians and extremum in the collected data.

However, chemotaxis now seems to overtake anemotaxis and gas-mapping in terms of accuracy, suggesting that it might be a good choice for teleoperated GSL that requires reliability and simplicity over precision.

\section{Search Efficiency}

The search efficiency $\eta$ is an index that should reveal if a low success ratio $(S)$ really means being unable to properly locate the gas source, or on the other hand, indicates that a particular search strategy trades accuracy for energy saving (i.e. provides coarser localization estimations but in shorter times).

Fig. 4 illustrates in a boxplot the values of this index for each scenario and GSL strategy. It can be seen that for a given scenario all strategies behave similarly (close medians and quartiles), which is a quite noteworthy result: there is no apparent correlation between accuracy and efficiency (see Fig. 5). This contradicts our initial intuition, which was that strategies that lead the operator to invest more search effort (i.e more time and therefore less efficiency) would have gotten much closer to the emission source (i.e higher accuracy). As can be seen, none of the compared

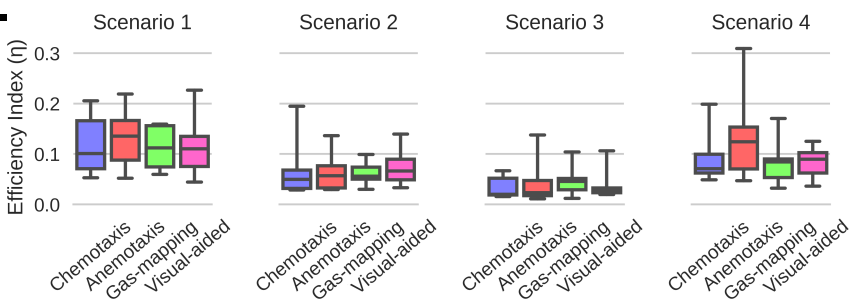

Fig. 4: Efficiency index for each scenario and GSL strategy. Efficiency measures the relation between the shortest possible path and the user's path.

search strategies shows this tendency, implying that the maximum accuracy of a given search strategy is limited by the environmental conditions.

\section{Search Behaviour}

Regarding how GSL strategies influence how the operators moved the robot while searching, we analyze next the most visited locations for the tested strategies and scenarios.

Fig. 6 depicts this information by plotting a heat map of where the operators spent their search time. For convenience, it also shows the gas distribution after the experiments' initial 60 seconds (first column), and the user declared gas source locations (i.e. the robot's end positions). As can be observed in all scenarios, the users tended to move along the center of the rooms and doorways for most part of the experiment, and expanded their search in proximity of the emission source or places with high gas concentrations. Still, there appear to be characteristic differences for each configuration:

- Chemotaxis appears to be the most exploratory of all strategies, probably because the users had to locate an initial trail of the gas distribution, and once they had found it, they also needed to determine its gradient.

- Anemotaxis reduces exploration by discarding rooms with clean air currents, and by instantly revealing the gross travel direction of gas plumes. However, the wind information appeared to confuse the users once in proximity of the emission source (particularly for Scenario 1), as vortices and turbulences were constantly stirring gas patches around.

- Gas mapping shows no notable differences with anemotaxis, despite that the employed predictive gas-mapping tool should have aided establishing a coarse location of the emission source.

- Visual-aided search clearly diverges from the other strategies in that it does not start with a search for a hint of the gas plume, but a direct approach towards the visible gas source candidates. As somehow expected, visual clues predominate remarkably over all other information sources, encouraging most operators to visit many false candidates and only stopping their search once they measured a high gas concentration in the proximity of one of them. As illustrated in Table I, this behaviour is not always optimum (e.g. Scenario 4), as it leads to the declaration of false emission candidates with high gas 
Authors' accepted manuscript: European Conference on Mobile Robotics (ECMR), 2017.

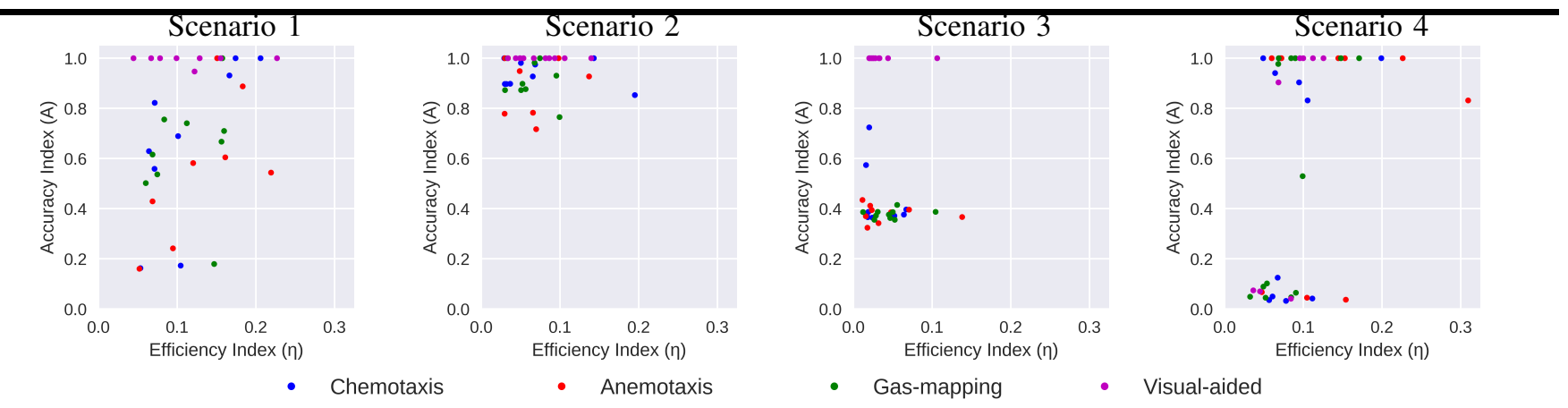

Fig. 5: Scatter plot comparing each experiment's accuracy index $(A)$ against if performance index ( $\eta$ ), grouped by test scenario and color coded to indicate the employed GSL strategy. For both axes, higher values are better (i.e. closer to the top right corner), indicating that the search was more efficient and accurate.
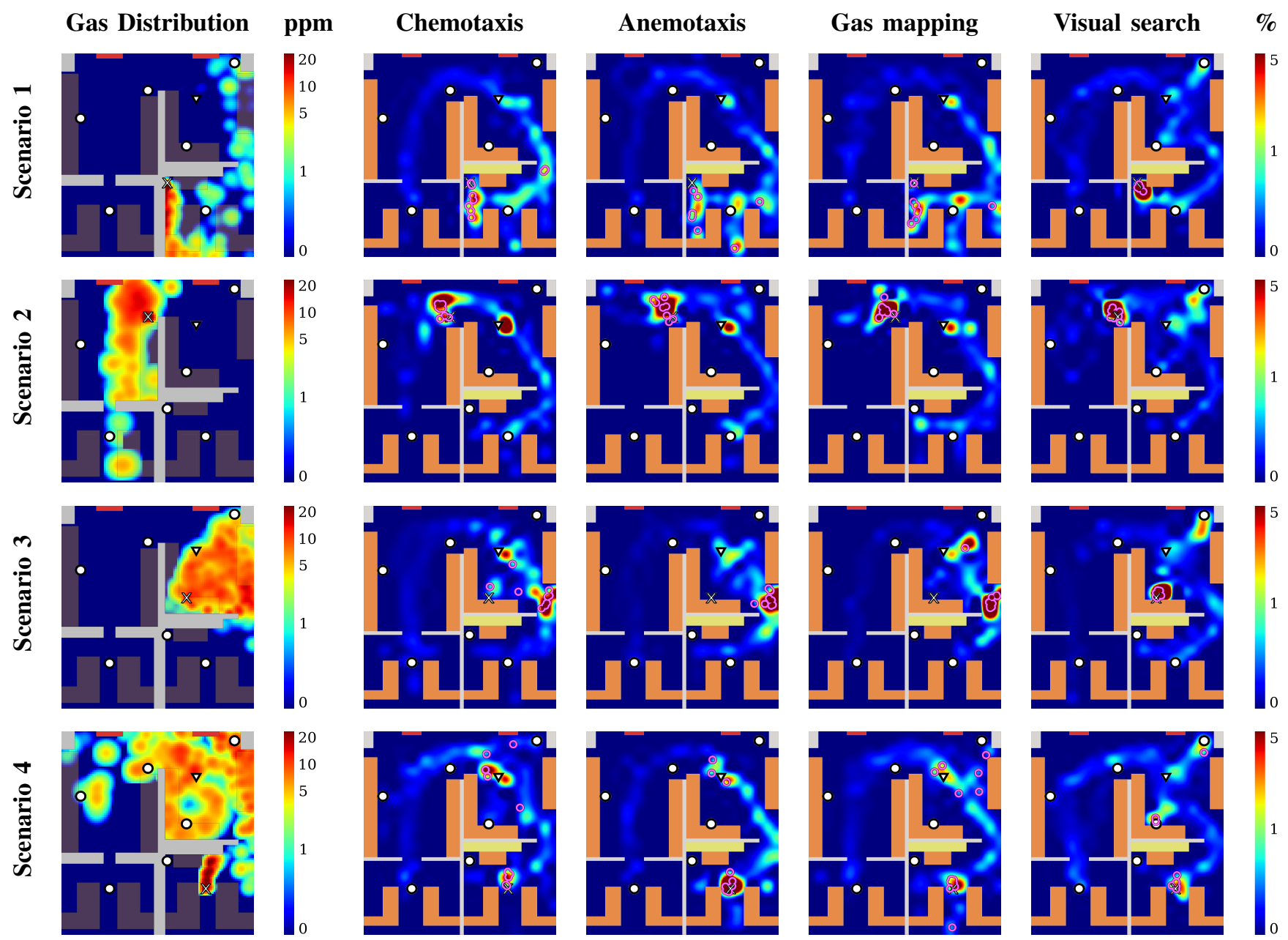

Fig. 6: Heat maps showing where the users (i.e. the robots) searched during the experiments, plotted as percent of the amount of time spent at any location for each strategy and scenario, and smoothed with a $0.5 \mathrm{~m}$ radius for visualization purposes. The robot's starting position is depicted as a triangle, the gas sources are shown as white crosses (active gas-leaks) and white circles (possible candidates shown during visual search), and the user declared gas source locations (i.e. the robot's end position) as pink circles. The environment's inner walls are shown in gray, and the furniture (not passable by the robot) in brown. For convenience, the left column also shows the gas distribution after the experiments' initial 60 seconds. Please note that the gas distributions in the experiment were dynamic, and therefore differed over time from those shown here. 
concentrations, just because the wind conditions where such that gas accumulated around them.

Conclusively, a GSL teleoperator would certainly benefit from training to take all information sources into account without being overwhelmed, and get the most out of wind and gas-map information even when presented with visual clues.

\section{CONClusions And Future Work}

In this work we have compared chemotaxis, anemotaxis, gas-mapping and visual-aided GSL strategies for telerobotics by analyzing a previously gathered dataset that contains more than 150 test-runs under different environmental conditions and gas distributions. We have evaluated their average effectiveness, accuracy, efficiency and overall impact on the operators with several performance indices, specifically proposed to deal with data coming from experiments with different test conditions, and resolved the following:

- Visual-aided search is the most effective GSL strategy, but it offers no advantages in terms of efficiency and can confuse the operator if various candidates have measurable gas around them.

- Chemotaxis is the best choice for applications that benefit from reliability and simplicity rather than precision.

- Anemotaxis and gas-mapping stand-out similarly at the beginning of the GSL process, but require trained operators to be effective once in close proximity to the emission source.

- There is no apparent correlation between accuracy and efficiency. How close the operators can get to the emission source only depends on the environmental conditions and the employed strategy.

For future research we plan to perform similar experiments in a real environment, despite a more limited control over the distribution of the emitted gas, to verify the results we have obtained so far. Subsequently, our long-term goals are to develop a fully functional platform for teleoperated GSL to research the human olfaction reasoning (i.e. how humans search for gas sources), and implement it in autonomous mobile robots.

\section{REFERENCES}

[1] T. Fong and C. Thorpe, "Vehicle teleoperation interfaces," Autonomous robots, vol. 11, no. 1, pp. 9-18, 2001.

[2] L. Srinivasavaradhan, G. Chandramouli, and A. Maniprashanna, "7 th sense. a multipurpose robot for military," in Perspective Technologies and Methods in MEMS Design, 2009. MEMSTECH 2009. 2009 5th International Conference on. IEEE, 2009, pp. 158-160.

[3] T. Kamegawa, T. Yarnasaki, H. Igarashi, and F. Matsuno, "Development of the snake-like rescue robot" kohga"," in Robotics and Automation, 2004. Proceedings. ICRA'04. 2004 IEEE International Conference on, vol. 5. IEEE, 2004, pp. 5081-5086.

[4] P. Pyk, S. B. i Badia, U. Bernardet, P. Knüsel, M. Carlsson, J. Gu, E. Chanie, B. S. Hansson, T. C. Pearce, and P. F. Verschure, "An artificial moth: Chemical source localization using a robot based neuronal model of moth optomotor anemotactic search," Autonomous Robots, vol. 20, no. 3, pp. 197-213, 2006.

[5] M. Vergassola, E. Villermaux, and B. I. Shraiman, “'infotaxis' as a strategy for searching without gradients," Nature, vol. 445, no. 7126, pp. 406-409, 2007.
[6] B. I. Shraiman and E. D. Siggia, "Scalar turbulence," Nature, vol. 405 , no. 6787, pp. 639-646, 2000.

[7] G. Ferri, E. Caselli, V. Mattoli, A. Mondini, B. Mazzolai, and P. Dario, "SPIRAL: A novel biologically-inspired algorithm for gas/odor source localization in an indoor environment with no strong airflow," Robotics and Autonomous Systems, vol. 57, no. 4, pp. 393-402, 2009.

[8] Y. Wada, H. Matsukura, and H. Ishida, "Estimation of gas source location from fluctuating readings of gas sensors and anemometer on mobile robot in outdoor environment," ECS Transactions, vol. 75, no. 16, pp. 99-106, 2016.

[9] C. Sanchez-Garrido, J. Monroy, and J. Gonzalez-Jimenez, "A configurable smart e-nose for spatio-temporal olfactory analysis," in IEEE Sensors, 2014, pp. 1968-1971.

[10] O. Rochel, D. Martinez, E. Hugues, and F. Sarry, "Stereo-olfaction with a sniffing neuromorphic robot using spiking neurons," in 16th European Conf. on Solid-State Transducers, 2002, pp. 4-p.

[11] H. Ishida, "Robotic systems for gas/odor source localization: Gap between experiments and real-life situations," in Proc. IEEE Int. Conf. on Robotics and Automation, 2007. (ICRA 2007), 2007, pp. 3-8.

[12] L. Marques, U. Nunes, and A. T. de Almeida, "Olfaction-based mobile robot navigation," Thin solid films, vol. 418, no. 1, pp. 51-58, 2002.

[13] J.-G. Li, Q.-H. Meng, Y. Wang, and M. Zeng, "Odor source localization using a mobile robot in outdoor airflow environments with a particle filter algorithm," Autonomous Robots, vol. 30, no. 3, pp. 281-292, 2011.

[14] P. P. Neumann, V. Hernandez Bennetts, A. J. Lilienthal, M. Bartholmai, and J. H. Schiller, "Gas source localization with a micro-drone using bio-inspired and particle filter-based algorithms," Advanced Robotics, vol. 27 , no. 9, pp. 725-738, 2013.

[15] J. Monroy, J.-L. Blanco, and J. Gonzalez-Jimenez, "Time-variant gas distribution mapping with obstacle information," Autonomous Robots, vol. 40, no. 1, pp. 1-16, 2016.

[16] J. Monroy, J. Gonzalez-Jimenez, and C. Sanchez-Garrido, "Monitoring household garbage odors in urban areas through distribution maps," in IEEE Sensors, 2014, pp. 1364-1367.

[17] M. Saska, J. Langr, and L. Přeučil, "Plume tracking by a selfstabilized group of micro aerial vehicles," in International Workshop on Modelling and Simulation for Autonomous Systems. Springer, 2014, pp. 44-55.

[18] H. Ishida, H. Tanaka, H. Taniguchi, and T. Moriizumi, "Mobile robot navigation using vision and olfaction to search for a gas/odor source," in Intelligent Robots and Systems, 2004.(IROS 2004). Proc. 2004 IEEE/RSJ Int. Conf., vol. 1. IEEE, 2004, pp. 313-318.

[19] P. Jiang, Q.-H. Meng, and M. Zeng, "Mobile robot gas source localization via top-down visual attention mechanism and shape analysis," in Intelligent Control and Automation (WCICA), 2010 8th World Congress on. IEEE, 2010, pp. 1818-1823.

[20] H. Ishida, T. Ushiku, S. Toyama, H. Taniguchi, and T. Moriizumi, "Mobile robot path planning using vision and olfaction to search for a gas source," in Sensors, 2005 IEEE. IEEE, 2005, pp. 4-pp.

[21] A. Gongora, J. Monroy, and J. Gonzalez-Jimenez, "A robotic experiment toward understanding human gas-source localization strategies,' in Int. Symposium on Olfaction and Electronic Nose (ISOEN), 2017.

[22] F. Melendez-Fernandez, C. Galindo, and J. Gonzalez-Jimenez, "A web-based solution for robotic telepresence," Submitted, 2017.

[23] J. Monroy, F. Melendez-Fernandez, A. Gongora, and J. GonzalezJimenez, "Integrating olfaction in a robotic telepresence loop," in Int. Symposium on Robot and Human Interactive Communication, 2017.

[24] Y. Zhang, Indoor air quality engineering. CRC press Boca Raton, FL, 2005.

[25] A. Lilienthal, H. Ulmer, H. Frohlich, A. Stutzle, F. Werner, and A. Zell, "Gas source declaration with a mobile robot," in Robotics and Automation, 2004. Proceedings. ICRA'04. 2004 IEEE International Conference on, vol. 2. IEEE, 2004, pp. 1430-1435.

[26] A. J. Lilienthal, T. Duckett, H. Ishida, and F. Werner, "Indicators of gas source proximity using metal oxide sensors in a turbulent environment," in Biomedical Robotics and Biomechatronics, 2006. BioRob 2006. The First IEEE/RAS-EMBS International Conference on. IEEE, 2006, pp. 733-738.

[27] A. T. Hayes, A. Martinoli, and R. M. Goodman, "Distributed odor source localization," IEEE Sensors Journal, vol. 2, no. 3, pp. 260$271,2002$. 